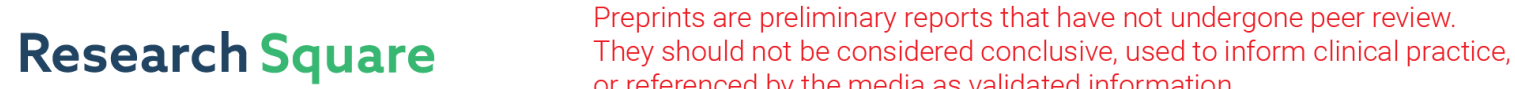 or referenced by the media as validated information. \\ Influence of Febrile Urinary Tract Infection on Ultrasonic Measurements of Nonreflux Upper Urinary Tract Dilation in Infants
}

\section{Peiqiang Li}

The Third Affiliated Hospital of Zhengzhou Universtiy

Fuyun Liu ( $\sim$ liufuyun111@126.com )

The Third Affiliated Hospital of Zhengzhou University

\section{Yan Huang}

The Third Affiliated Hospital of Zhengzhou Universtiy

\section{Research}

Keywords: Anteroposterior renal pelvic diameter, urinary tract infection, hydronephrosis, ureterovesical junction obstruction, ureteropelvic junction obstruction

Posted Date: February 23rd, 2021

DOI: https://doi.org/10.21203/rs.3.rs-231568/v1

License: (c) (i) This work is licensed under a Creative Commons Attribution 4.0 International License. Read Full License 


\section{Abstract}

Background To investigate the changes in ultrasonic measurements of nonreflux upper urinary tract dilation in infants with febrile urinary tract infection (UTI).

Methods There were 28 cases of nonreflux upper urinary tract dilatation with febrile UTI: 14 cases of ureteropelvic junction obstruction (UPJO) (14 kidneys) and 14 cases of ureterovesical junction obstruction (UVJO) (16 kidneys). Changes in anteroposterior renal pelvic diameter (APD) and ureteral dilatation during infection and after infection were compared in UPJO and UVJO patients, respectively.

Results In the UPJO with febrile UTI group, the APD was $24.1 \pm 10.0 \mathrm{~mm}$ at the time of UTI and $16.6 \pm 7.0$ $\mathrm{mm} 1$ week after infection recovery $(P<0.001)$. In the UVJO with febrile UTI group, the APD was $19.3 \pm 8.5$ $\mathrm{mm}$ at the time of UTI and $15.2 \pm 7.7 \mathrm{~mm} 1$ week after infection recovery $(P<0.001)$. In the UVJO with febrile UTI group, the ureteric diameter was $11.0 \pm 3.2 \mathrm{~mm}$ during UTI and $6.8 \pm 2.6 \mathrm{~mm} 1$ week after infection recovery $(P<0.001)$.

Conclusions In UPJO patients, the APD decreased after febrile UTI treatment compared with that during infection. In UVJO patients, the APD and ureteric diameter decreased after febrile UTI treatment compared with that during infection.

\section{Introduction}

In infants, upper urinary tract dilatation due to upper urinary tract obstruction affects kidney function and can lead to urinary tract infection (UTI) if progression continues(1-3). The first two most common causes of upper urinary tract dilatation in infants are ureteropelvic junction obstruction (UPJO) and ureterovesical junction obstruction (UVJO)(2). Ultrasound is a commonly used follow-up method $(2,4,5)$. One surgical indication for upper urinary tract dilatation is an increase in the anteroposterior renal pelvic diameter (APD) or ureteric diameter during follow-up(2). One animal experiment suggested that ureteral contraction could be reversibly inhibited by bacteria in the ureteral lumen(6). Another animal study demonstrated that the combination of infection and obstructive hydronephrosis caused renal pelvic pressure elevation that was higher than that associated with either infection or obstructive hydronephrosis alone(7). Upper UTIs often have systemic symptoms such as fever(8-10). Our purpose is to investigate the changes in ultrasonic measurements of nonreflux upper urinary tract dilation in infants with febrile UTI.

\section{Patients And Methods}

Retrospective analysis was performed on cases of patients with nonreflux upper urinary tract dilatation combined with febrile UTI who were followed and treated in our hospital from June 2015 to June 2020. The exclusion criteria were dysplastic or hyperechogenic kidney, megaureter caused by vesicoureteral reflux, posterior urethral valves, and neurogenic bladder. All patients were uncircumcised. 
Patients were divided into UPJO and UVJO groups. Changes in the APD and ureteral dilatation during infection and after infection were compared in the two groups.

Ultrasonic examination of the urinary system was performed in the supine position after adequate oral hydration. The APD was defined as the diameter at which the kidney was measured in the transverse plane at the mid-level of the kidney(11). The upper, middle and lower ureteric diameters were measured, and the mean values of the three values were taken. Measurements were taken once during and once after infection. Hydration status, bladder filling, and operator skills influence the results of ultrasound measurements(5). All ultrasound measurements were performed by the same well-trained sonographer. The timing of measurement was when the bladder was full after oral liquid intake (water, milk or juice) at $15 \mathrm{ml} / \mathrm{kg}$ body weight(12).

The diagnosis of febrile UTI included the following criteria: fever exceeding 38 degrees Celsius, pyuria (positive leukocyte esterase, greater than 5 white blood cells per high-power field) and urine culture $\left(>10^{5}\right.$ $\mathrm{cfu} / \mathrm{mL}$ of voided urine of a single microorganism in a specimen collected by clean-catch urine collection)(2).

Intravenous antibiotics and ultrasound examinations were performed immediately after the diagnosis of febrile UTI. Ultrasound examination and voiding cystourethrography were performed one week after the body temperature was normal, pyuria disappeared, and urine bacteria culture was negative.

\section{Statistical analysis}

The K-S test was used to test normality. Spearman rank correlation analysis was used to analyze the correlation of data. The difference in the first and second ultrasonic measurement results was analyzed by paired-samples T tests. SPSS version 26 was used for statistical analysis. P values less than 0.05 were considered statistically significant.

\section{Results}

\section{Patient characteristics}

A total of 14 febrile UTIs occurred in 289 follow-up UPJO patients. Age range: 1 month to 4 years, median: 7 months ( 3 months to 2 years and 10 months old, interquartile range). All 14 patients had unilateral hydronephrosis in a total of 14 kidneys. There were 2 patients with grade I vesicoureteral reflux, and no ureteral dilatation was measured by ultrasound.

A total of 14 febrile UTIs occurred in 67 follow-up UVJO patients. Age range: 2 months to 5 years, median: 11 months (4 months to 1 year and 11 months old, interquartile range). There were 2 patients with bilateral hydroureteronephrosis and bilateral infections in a total of 16 kidneys. 


\section{Ultrasound outcomes}

In the UPJO with febrile UTI group, the APD was $24.1 \pm 10.0 \mathrm{~mm}$ at the time of UTI and $16.6 \pm 7.0 \mathrm{~mm} 1$ week after infection recovery $(P<0.001)$ (paired-samples $T$ test). No abnormal ureteral dilatation was measured on ultrasound at the time of UTI or after infection recovery. The time distance between the first and second measurements was $12.4 \pm 1.5$ days (mean $\pm S D$ ). Age was not associated with the APD at the time of infection (Spearman rank correlation analysis, $\mathrm{P}=0.307$ ). The raw data are shown in Table 1 .

In the UVJO with febrile UTI group, the APD was $19.3 \pm 8.5 \mathrm{~mm}$ at the time of UTI and $15.2 \pm 7.7 \mathrm{~mm} 1$ week after infection recovery $(\mathrm{P}<0.001)$ (paired-samples $T$ test). The ureteric diameter was $11.0 \pm 3.2 \mathrm{~mm}$ during urinary tract infection and $6.8 \pm 2.6 \mathrm{~mm} 1$ week after infection recovery $(P<0.001)$. The time distance between the first and second measurements was 12.2 \pm 1.5 days (mean $\pm S D$ ). Age was not associated with the APD or ureteric diameter at the time of infection (Spearman rank correlation analysis, $P=0.382, P=0.271)$. The raw data are shown in Table 2 .

The paired sample T-test results of the first and second ultrasonic measurements are summarized in Table 3. 
Table 1

Ultrasound outcomes in the UPJO group

\begin{tabular}{|lllll|}
\hline No. & Sex & Age (months) & APD (mm) & \\
\cline { 3 - 4 } & & & When the febrile UTls & UTls were cured \\
\hline 1 & Male & 12 & 35 & 29 \\
\hline 2 & Male & 3 & 24 & 15 \\
\hline 4 & Male & 32 & 32 & 20 \\
\hline 5 & Male & 3 & 13 & 13 \\
\hline 6 & Male & 9 & 33 & 27 \\
\hline 7 & Male & 39 & 30 & 17 \\
\hline 8 & Female & 48 & 10 & 5 \\
\hline 9 & Female & 1 & 39 & 13 \\
\hline 10 & Male & 3 & 21 & 18 \\
\hline 11 & Male & 7 & 20 & 5 \\
\hline 12 & Female & 40 & 11 & 17 \\
\hline 13 & Male & 7 & 19 & 20 \\
\hline 14 & Female & 6 & 36 & 12 \\
\hline normality test (K-S test) P & 0.002 & 14 & 0.200 \\
\hline $\begin{array}{l}\text { APD anteroposterior renal pelvic diameter. UPJO ureteropelvic junction obstruction. UTI urinary tract } \\
\text { infection. }\end{array}$ & & & \\
\hline
\end{tabular}


Table 2

Ultrasound outcomes in the UVJO group

\begin{tabular}{|c|c|c|c|c|c|c|}
\hline \multirow[t]{2}{*}{ No. } & \multirow[t]{2}{*}{ Sex } & \multirow{2}{*}{$\begin{array}{l}\text { Age } \\
\text { (months) }\end{array}$} & \multicolumn{2}{|l|}{ APD (mm) } & \multicolumn{2}{|c|}{ Ureteric diameter (mm) } \\
\hline & & & $\begin{array}{l}\text { When the } \\
\text { febrile UTIs }\end{array}$ & $\begin{array}{l}\text { UTIs were } \\
\text { cured }\end{array}$ & $\begin{array}{l}\text { When the } \\
\text { febrile UTIs }\end{array}$ & $\begin{array}{l}\text { UTIs were } \\
\text { cured }\end{array}$ \\
\hline 1 & Male & 51 & 26 & 19 & 11 & 7 \\
\hline 2 & Male & 15 & 24 & 16 & 7 & 5 \\
\hline 3 & Female & 3 & 22 & 19 & 9 & 6 \\
\hline 4 & Male & 11 & 16 & 10 & 9 & 5 \\
\hline 5 & Male & 14 & 19 & 17 & 15 & 13 \\
\hline 6 & Female & 45 & 8 & 7 & 14 & 6 \\
\hline $7 *$ & Male & 11 & 12 & 8 & 13 & 7 \\
\hline $8^{*}$ & Male & 11 & 10 & 7 & 12 & 8 \\
\hline 9 & Male & 2 & 14 & 11 & 7 & 2 \\
\hline 10 & Male & 10 & 30 & 28 & 14 & 8 \\
\hline $11^{\star \star}$ & Male & 3 & 22 & 13 & 5 & 5 \\
\hline $12^{\star \star}$ & Male & 3 & 13 & 8 & 11 & 7 \\
\hline 13 & Male & 15 & 22 & 17 & 7 & 4 \\
\hline 14 & Female & 60 & 6 & 7 & 14 & 7 \\
\hline 15 & Male & 4 & 33 & 27 & 14 & 11 \\
\hline 16 & Male & 8 & 32 & 29 & 14 & 8 \\
\hline \multicolumn{2}{|c|}{$\begin{array}{l}\text { normality test (K-S } \\
\text { test) } P\end{array}$} & $<0.001$ & 0.200 & 0.200 & 0.096 & 0.088 \\
\hline \multicolumn{7}{|c|}{$\begin{array}{l}\text { Numbers } * 7 \text { and } 8 \text { and } * * 11 \text { and } 12 \text { are bilateral ultrasound measurements from the same patients, } \\
\text { respectively. }\end{array}$} \\
\hline
\end{tabular}


Table 3

Paired-sample T test was performed on the results of the first and second ultrasound measurements

$\begin{array}{llll}\text { Characteristics } \quad \text { When the febrile UTIs } & \text { UTls were cured }\end{array}$

UPJO (14 kidneys)
APD
$24.1 \pm 10.0 \mathrm{~mm}$
$16.6 \pm 7.0 \mathrm{~mm}$
$<0.001$

UVJO (16 kidneys)

APD

$19.3 \pm 8.5 \mathrm{~mm}$

$15.2 \pm 7.7 \mathrm{~mm}$

$<0.001$

Ureteric diameter

$11.0 \pm 3.2 \mathrm{~mm}$

$6.8 \pm 2.6 \mathrm{~mm}$

$<0.001$

Values are expressed as the mean \pm SD. APD anteroposterior renal pelvic diameter. UPJO ureteropelvic junction obstruction. UVJO ureterovesical junction obstruction. UTI urinary tract infection.

The bacterial culture results of 28 cases were as follows: 9 cases of Escherichia coli, 5 cases of Klebsiella pneumoniae, 3 cases of Acinetobacter baumannii, 2 cases of Enterobacter aerogenes, 2 cases of Acinetobacter calcoaceticus, 2 cases of Staphylococcus xylosus, 2 cases of Staphylococcus aureus, 2 cases of Klebsiella ozaenae, and 1 case of Enterobacter agglomerate.

\section{Discussion}

One animal experiment suggested that ureteral contraction could be reversibly inhibited by bacteria in the ureteral lumen(6). Moreover, $55-70 \%$ of spontaneous rhythmic contractions in sheep ureters were inhibited by the addition of small amounts of growth supernatants from E. coli, Pseudomonas aeruginosa and Klebsiella pneumoniae(13). In our observation, in the case of UVJO combined with febrile UTIs, the degree of ureteral dilatation and the APD during UTI were both higher than the values one week after infection recovery. Ureteropelvic dilatation was relieved after the infection was cured. Similar results were observed in clinical patients and in animal experiments.

Another animal study demonstrated that the combination of infection and obstructive hydronephrosis caused renal pelvic pressure elevation that was higher than that associated with either infection or obstructive hydronephrosis alone(7). In our observation, in the case of UPJO combined with febrile UTIs, the degree of APD during UTI was higher than the values one week after infection recovery. The effect of UTI on the aggravation of hydronephrosis due to UPJO has been demonstrated in clinical patients. However, in the case of UPJO, only the influence of infection on the APD was observed, while the ureter, which was not originally dilated, did not expand under the influence of infection.

There are studies on the mechanism of UTI affecting the upper urinary tract. Flagella and flagellummediated motility/chemotaxis contribute to the fitness of uropathogenic E. coli and therefore significantly enhance the pathogenesis of UTIs caused by uropathogenic E. coli (14). E. coli impair ureteric contractility in a Ca-dependent manner, largely caused by the stimulation of potassium channels, and this 
mechanism is dependent on host-urothelium interactions(15). The second messenger $\mathrm{Ca}^{2+}$ activates the $\mathrm{Ca}^{2+} /$ calmodulin-dependent myosin light chain kinase-dependent phosphorylation of 20-kDa regulatory light chains of myosin, which leads to ureteric contraction. Inflammatory factors can initiate spontaneous activity in the proximal and distal ureter(16).

Many animal experiments and mechanisms of infection inhibiting ureteral peristalsis and causing urinary tract dilatation have been studied. The novelty of this study is that urinary tract expansion of UPJO or UVJO patients complicated by febrile UTIs was found to be lower after the infection was cured than during the infection. The clinical significance is described below.

When UPJO or UVJO leads to dilatation of the upper urinary tract, some patients may heal spontaneously, while others may require surgery. One of the surgical indications is a progressive increase in upper urinary tract hydronephrosis during follow-up(2). Febrile UTI is unpleasant but can be quickly controlled with accurate diagnosis and timely treatment. In patients with febrile UTIs, the dilatation of the upper urinary tract was reduced after antibacterial treatment. Therefore, if febrile UTI is present, the influence of UTI should be taken into account in the evaluation of the degree of upper urinary tract dilation to make a more comprehensive surgical decision.

It would be even better to obtain ultrasound values of hydronephrosis within a short period of time (e.g., 1 week) before febrile UTI. We can analyze the dynamic changes in ultrasound results before, during, and after febrile urinary tract infection. However, it is impossible to precisely predict, in advance, which patients will develop febrile UTI. This is clinically challenging. In addition, this was a single-center retrospective study, and the number of single-center cases was not large. If prospective studies can be conducted to obtain the degree of upper urinary tract dilation before, during, and after infection recovery and to obtain more cases, the influence of urinary tract infection on upper urinary tract dilation will be further clarified.

\section{List Of Abbreviations}

UTI, urinary tract infection

UPJO , ureteropelvic junction obstruction

UVJO, ureterovesical junction obstruction

APD , anteroposterior renal pelvic diameter

\section{Declarations}

\section{Funding}

This study was funded by Health Commission of Henan Province (Number LHGJ20190383). 


\section{Conflicts of interest}

The authors have no financial or proprietary interests in any material discussed in this article.

\section{Authors' contributions}

Peiqiang Li conceptualized and designed the study, drafted the initial manuscript. Peiqiang Li, Yan Huang, and Fuyun Liu carried out the initial analyses, reviewed, and revised the manuscript. Peiqiang Li supervised the statistics. Fuyun Liu made critical revision of the manuscript for important intellectual content. All authors approved the final manuscript as submitted and agree to be accountable for all aspects of the work.

\section{Ethics approval}

Ethical approval was waived by the local Ethics Committee of The Third Affiliated Hospital of Zhengzhou University in view of the retrospective nature of the study, and all the procedures performed were part of routine care.

\section{Consent to participate}

Informed consent was obtained from the legal guardians.

\section{The statement}

All the experimental protocols for the involvement of human data in the study were in accordance with the principles of the Declaration of Helsinki.

\section{References}

1. Coelho GM, Bouzada MCF, Lemos GS, Pereira AK, Lima BP, Oliveira EA. Risk factors for urinary tract infection in children with prenatal renal pelvic dilatation. J Urol. 2008;179(1):284-9.

2. Radmayr C, Bogaert G, Dogan HS, Nijman R, Silay MS, Stein R, et al. EAU Guidelines on Paediatric Urology 2020. European Association of Urology Guidelines 2020 Edition. presented at the EAU Annual Congress Amsterdam 2020. Arnhem, The Netherlands: European Association of Urology Guidelines Office; 2020.

3. Kohno M, Ogawa T, Kojima Y, Sakoda A, Johnin K, Sugita Y, et al. Pediatric congenital hydronephrosis (ureteropelvic junction obstruction): Medical management guide. Int J Urol. 2020;27(5):369-76.

4. Rianthavorn P, Limwattana S. Diagnostic accuracy of neonatal kidney ultrasound in children having antenatal hydronephrosis without ureter and bladder abnormalities. World J Urol. 2015;33(10):164550 .

5. Nguyen HT, Herndon CDA, Cooper C, Gatti J, Kirsch A, Kokorowski P, et al. The Society for Fetal Urology consensus statement on the evaluation and management of antenatal hydronephrosis. $\mathrm{J}$ 
Pediatr Urol. 2010;6(3):212-31.

6. Lennon GM, Ryan PC, Fitzpatrick JM. THE URETER IN-VITRO - NORMAL MOTILITY AND RESPONSE TO URINARY PATHOGENS. Br J Urol. 1993;72(3):284-90.

7. Hansen MH, Wang BY, Afzal N, Boineau FG, Lewy JE, Shortliffe LMD. Effect of urinary tract infection on ureteropelvic junction obstruction in a rat model. Urology. 2003;61(4):858-63.

8. Roberts KB, Downs SM, Finnell SME, Hellerstein S, Shortliffe LD, Wald ER, et al. Urinary Tract Infection: Clinical Practice Guideline for the Diagnosis and Management of the Initial UTI in Febrile Infants and Children 2 to 24 Months. Pediatrics. 2011;128(3):595-610.

9. Shaikh N, Mattoo TK, Keren R, Ivanova A, Cui G, Moxey-Mims M, et al. Early Antibiotic Treatment for Pediatric Febrile Urinary Tract Infection and Renal Scarring. Jama Pediatrics. 2016;170(9):848-54.

10. Karavanaki KA, Soldatou A, Koufadaki AM, Tsentidis C, Haliotis FA, Stefanidis CJ. Delayed treatment of the first febrile urinary tract infection in early childhood increased the risk of renal scarring. Acta Paediatr. 2017;106(1):149-54.

11. Chitty LS, Altman DG. Charts of fetal size: kidney and renal pelvis measurements. Prenat Diagn. 2003;23(11):891-7.

12. Majd M, Bar-Sever Z, Santos AI, De Palma D. The SNMMI and EANM Procedural Guidelines for Diuresis Renography in Infants and Children. J Nucl Med. 2018;59(10):1636-40.

13. Thulesius O, Araj G. The effect of uropathogenic bacteria on ureteral motility. Urol Res. $1987 ; 15(5): 273-6$.

14. Lane MC, Lockatell V, Monterosso G, Lamphier D, Weinert J, Hebel JR, et al. Role of motility in the colonization of uropathogenic Escherichia coli in the urinary tract. Infection and Immunity. 2005;73(11):7644-56.

15. Floyd RV, Winstanley C, Bakran A, Wray S, Burdyga TV. Modulation of ureteric Ca signaling and contractility in humans and rats by uropathogenic E. coli. Am J Physiol-Renal Physiol. 2010;298(4):F900-F8.

16. Burdyga T, Lang RJ. Excitation-Contraction Coupling in Ureteric Smooth Muscle: Mechanisms Driving Ureteric Peristalsis. Smooth Muscle Spontaneous Activity: Physiological and Pathological Modulation. 2019;1124:103-19. 